\title{
Rigidity for the Hyperbolic Monge-Ampère Equation
}

\author{
CHUN-CHI LIN
}

\begin{abstract}
Some properties of nonlinear partial differential equations are naturally associated with the geometry of sets in the space of matrices. In this paper we consider the model case when the compact set $K$ is contained in the hyperboloid $\mathcal{H}_{-1}$, where $\mathcal{H}_{-1} \subset \mathbb{M}_{\text {sym }}^{2 \times 2}$, the set of symmetric $2 \times 2$ matrices. The hyperboloid $\mathcal{H}_{-1}$ is generated by two families of rank-one lines and related to the hyperbolic Monge-Ampère equation det $\nabla^{2} u=-1$. For some compact subsets $K \subset \mathcal{H}_{-1}$ containing a rank-one connection, we show the rigidity property of $K$ by imposing proper topology in the convergence of approximate solutions and affine boundary conditions.
\end{abstract}

Mathematics Subject Classification (2000): 49J10 (primary); 74G65, 35L70 (secondary).

\section{1. - Introduction}

This paper is motivated by the rigidity problems studied by Chlebík and Kirchheim in [5] and [15]. In connection with mathematical models of crystalline microstructures ([2], [6], [7]), one is interested in exact and approximate solutions of the partial differential inclusions (relations) $\nabla f \in K$ a.e. in $\Omega$, where $f: \Omega \subset \mathbb{R}^{n} \rightarrow \mathbb{R}^{m}$ is a Lipschitz map on a bounded domain $\Omega$ and $K$ is a compact set of matrices in $\mathbb{M}^{m \times n}$. We say that the compact set $K$ is rigid for exact solutions if each Lipschitz map $f$ with $\nabla f \in K$ a.e. in $\Omega$ is necessarily an affine map. The compact set $K$ is rigid for approximate solutions if, for each convergent subsequence of Lipschitz maps $f^{(j)}$ with $\operatorname{dist}\left(\nabla f^{(j)}, K\right) \rightarrow 0$ in measure in $\Omega$, the limit map $f$ is necessarily an affine map. One of the famous rigidity problems is the one-well problem (i.e., $K=\mathrm{SO}(n), m=n$ ) in [14]. The other one is the $N$-gradient problem for $N \in\{2,3,4\}$ (i.e., $K$ consists of $N$ points in $\mathbb{M}^{m \times n}$ ) in [2], [24], [5]. These types of (geometric) rigidity properties for the mappings are useful and have many important applications, for example in general elasticity theroy (e.g. [13]) or deriving plate theory from 3-dimensional elasticity (see [11]). 
None of the sets considered in [14], [2], [24] and [5] contains a rankone connection. A set $K$ is said to contain a rank-one connection if there are two matrices $A, B \in K$ satisfying $\operatorname{rk}(A-B) \leq 1$. Whenever a set $K$ contains a rank-one connection, one may construct an exact or approximate solution such that $\nabla f$ oscillates strongly. In this situation, which corresponds to a loss of ellipticity, one cannot have a rigidity result for arbitrary solutions. However, the absence of a rank-one connection does not guarantee rigidity, i.e., the absence of microstructures (see [17] for the survey and examples). Especially, the so called $T_{4}$-configuration consisting of four matrices without a rank-one connection, which was observed independently in different contexts ([21], [1], [20], [3], [25]), has been applied to the construction of solutions of elliptic systems with nowhere $C^{1}$ (e.g., see [19]). This reveals the difficulty in deriving the regularity theory for general elliptic systems of PDEs. Thus, we raised the question: is certain rigidity property restored when proper topology in the convergence of approximate solutions and suitable (e.g. affine) boundary conditions are imposed? An interesting model case is to consider a compact subset $K$ of the set

$$
\mathcal{H}_{-D}:=\left\{M \in \mathbb{M}_{\text {sym }}^{2 \times 2}: \operatorname{det}(M)=-D\right\} \subset \mathbb{R}^{3}, D>0 .
$$

Using the coordinates $\left(\begin{array}{cc}Z+X & Y \\ Y & Z-X\end{array}\right)$ on the symmetric $2 \times 2$ matrices, we see that $\mathcal{H}_{-D}$ is a hyperboloid of one sheet in $\mathbb{R}^{3}$. It is generated by two families of straight lines which are exactly the rank-one lines on $\mathcal{H}_{-D}$. Through each point on $\mathcal{H}_{-D}$ there are exactly two rank-one lines. Thus, $\mathcal{H}_{-D}$ locally behaves in many ways like the linear space of diagonal $2 \times 2$ matrices, which is wellunderstood ([4], [18], [25]). However, $\mathcal{H}_{-D}$ globally exhibits much more subtle behaviour (see [15] and the discussion below).

In [5] and [15], Chlebík and Kirchheim have analyzed the behaviour of Lipschitz maps with $\nabla f \in \mathcal{H}_{-1}$, i.e.,

$$
\left\{\begin{array}{l}
(\nabla f)^{T}=\nabla f \\
\operatorname{det} \nabla f=-1
\end{array}\right.
$$

This is equivalent to the hyperbolic Monge-Ampère equation,

$$
\operatorname{det} \nabla^{2} u=-1 \text { in } \Omega, u \in W^{2, \infty}(\Omega),
$$

where $f=\nabla u$. This equation is locally determined by two functions of one variable. In fact, Equation (1.2) can be transformed into the linear wave equation by a nonlinear change of variables. To see this, assume at $x_{0} \in \Omega$, $\partial_{1}^{2} u\left(x_{0}\right) \neq 0$ and $\partial_{2}^{2} u\left(x_{0}\right) \neq 0$ (hence $\partial_{1} \partial_{2} u\left(x_{0}\right) \neq \pm 1$ ). This assumption can always be achieved by a suitable rotation of coordinates. Then, we will see in the following that we can use $\left(f_{2}(x)-x_{1}, f_{2}(x)+x_{1}\right)$ as independent variables 
and $\left(f_{1}(x)+x_{2}, f_{1}(x)-x_{2}\right)$ as dependent variables. Since $\nabla f$ is symmetric and $\operatorname{det} \nabla f=-1$, the map

$$
f^{-}(x):=f(x)-\left(\begin{array}{c}
-x_{2} \\
x_{1}
\end{array}\right)
$$

satisfies

$$
\operatorname{det} \nabla f^{-}=\operatorname{det}\left(\nabla f-\left(\begin{array}{cc}
0 & -1 \\
1 & 0
\end{array}\right)\right)=0 .
$$

Thus, $f_{1}(x)+x_{2}$ is locally a function of $f_{2}(x)-x_{1}$, by the implicit function theorem. Similar reasoning for

$$
f^{+}(x):=f(x)+\left(\begin{array}{c}
-x_{2} \\
x_{1}
\end{array}\right)
$$

shows that there exist locally two functions $d_{1}$ and $d_{2}$ such that near $x_{0}$,

$$
\left(\begin{array}{l}
f_{1}(x)+x_{2} \\
f_{1}(x)-x_{2}
\end{array}\right)=\left(\begin{array}{l}
d_{1}\left(f_{2}(x)-x_{1}\right) \\
d_{2}\left(f_{2}(x)+x_{1}\right)
\end{array}\right)
$$

To the author's knowledge, this method was first used by Heinz [12] for the elliptic Monge-Ampère equation. In Schulz's book [23], this method was called the Legendre-like transformation and applied to derive the regularity for the twodimensional elliptic Monge-Ampère equations. Further references concerning this method include [9], [22] for the elliptic case, and [16] for the hyperbolic case. For the standard Legendre transformation converting a nonlinear PDE into a linear one, readers are referred to Chapter 4 of [8]. Kirchheim in [15] gave an example showing that Equation (1.3) may fail for $W^{2, \infty}$ solutions of Equation (1.2) or, equivalently, $W^{1, \infty}$ solutions of Equation (1.1). However, he showed that the set of so called branch points $\bar{x}$, for which Equation (1.3) does not hold in a neighborhood of $\bar{x}$ (after suitable rotation of local coordinates), is discrete. Furthermore, for exact Lipschitz solutions of Equation (1.1) with affine boundary condition, Kirchheim proved that any compact subset $K \subset \mathcal{H}_{-1}$ is rigid.

In the following, we consider the perturbed equation of Equation (1.2),

$$
\operatorname{det} \nabla^{2} u=-\varphi^{2}
$$

for $u \in W^{2, \infty}(\Omega)$, where $\varphi^{2}$ means the square of a Lipschitz continuous function $\varphi$ with $\|\varphi-1\|_{W 1, \infty}$ sufficiently small. On the existence of $W^{2, \infty}$-solutions of Equation (1.4) with affine boundary data, the reader is refered to Chapter 3 in [15] (solving partial differential inclusions). However, the function $\varphi$ (or $\operatorname{det} \nabla^{2} u$ ) in these examples is only in $L^{\infty}$. Note, when $\Omega \subset \mathbb{R}^{2}$ is a bounded and simply connected domain, Equation (1.4) is over-determined as a PDE problem with Dirichlet or Neumann boundary condition. Here, we skip the 
issue of existence problem but only focus on the rigidity property for the type of Equation (1.4) on a bounded domain in $\mathbb{R}^{2}$, which is not necessarily simplyconnected. We show that a perturbed version of Equation (1.3) holds locally if the oscillation of $\nabla^{2} u$ is not very large (see Theorem 1). By applying the Legendre-like transformations, we establish the Bilipschitz maps $S, T, h$ between domains $\Omega, \mathcal{U}_{z}, \mathcal{U}_{\xi}$, shown in Figure 1 (see the definitions of $S, T, h$ and $\mathcal{U}_{z}, \mathcal{U}_{\xi}$ in Step $1^{0}$ of the proof of Theorem 1). Since the map $h\left(\right.$ or $\left.g=h^{-1}\right)$ satisfies the quasilinear hyperbolic $2 \times 2$ system, for a fixed point $z^{(0)} \in \mathcal{U}_{z}$ (or $\xi^{(0)}=$ $\left.h\left(z^{(0)}\right) \in \mathcal{U}_{\xi}\right)$ and $\pm \in\{+,-\}$, there are exactly two characteristic curves, or so called rarefaction curves, denoted by $R_{ \pm}\left(z^{(0)}\right)$ (or $E_{ \pm}\left(\xi^{(0)}\right)$ ), passing through $z^{(0)}$ (or $\left.\xi^{(0)}\right)$ with strictly positive and negative finite slopes. Notice that for each $\pm \in\{+,-\}$ we have $S^{-1}\left(R_{ \pm}\left(z^{(0)}\right)\right)=T^{-1}\left(E_{ \pm}\left(\xi^{(0)}\right)\right) \subset \Omega$, which intersects the lines $\left\{x_{1}=c_{1}\right\}$ and $\left\{x_{2}=c_{2}\right\}$ transversely for some $\left(c_{1}, c_{2}\right) \in \Omega$. Denote by $\left(c_{1}, w_{ \pm}\left(z^{(0)}\right)\right)=S^{-1}\left(R_{ \pm}\left(z^{(0)}\right)\right) \cap\left\{x_{1}=c_{1}\right\}$ and $\left(w_{ \pm}\left(\xi^{(0)}, c_{2}\right)\right)=T^{-1}\left(E_{ \pm}\left(\xi^{(0)}\right)\right) \cap$ $\left\{x_{2}=c_{2}\right\}$ the points of intersection in $\Omega$. Thus in Step $2^{0}$ of the proof of Theorem 1, for each point $x=S^{-1}(z)=T^{-1}(\xi) \in \Omega$, we can locally define the Bilipschitz maps $R: \mathcal{U}_{z} \rightarrow \mathbb{R}^{2}$ and $E: \mathcal{U}_{\xi} \rightarrow \mathbb{R}^{2}$ by $R(z)=\left(w_{+}(z), w_{-}(z)\right)$ and $E(\xi)=\left(\eta_{+}(\xi), \eta_{-}(\xi)\right)$. Since $w_{ \pm}(\widetilde{z})=w_{ \pm}(z)\left(\right.$ or $\left.\eta_{ \pm}(\widetilde{\xi})=\eta_{ \pm}(\xi)\right)$ whenever $\widetilde{z} \in$ $R_{ \pm}(z)$ (or $\widetilde{\xi} \in E_{ \pm}(\xi)$ ), we call $w_{ \pm}(z)$ (or $\eta_{ \pm}(\xi)$ ) the Riemann invariants for all $\pm \in\{+,-\}$, in order to be consistant with the terminology of hyperbolic systems in PDE theroy. We further define the Bilipschitz map $D: R\left(\mathcal{U}_{z}\right) \rightarrow E\left(\mathcal{U}_{\xi}\right)$ locally by $D\left(w_{+}(z), w_{-}(z)\right)=\left(\eta_{+}(\xi), \eta_{-}(\xi)\right)$. Since $\cup_{ \pm, z} S^{-1}\left(R_{ \pm}(z)\right)$ forms a local coordinate net in $\Omega$ and for each fixed \pm and $z, S^{-1}\left(R_{ \pm}(z)\right)$ intersects the straight lines $\left\{x_{1}=c_{1}\right\}$ and $\left\{x_{2}=c_{2}\right\}$ transversely, the Bilipschitz map $D$ is a diagonal map, i.e., $\nabla D$ is a diagonal $2 \times 2$ matrix. The above argument is only a local one. We extend it to be global by applying the Kirszbraun's Lipschitz extension theorem (see Federer [10] 2.10.43). The estimate in Corollary 1 is a direct consequence of the diagonal map $D$ and the affine boundary condition. For a nonconvex domain $\Omega$, we refine the above argument to derive the rigidity results for some compact subsets of $\mathcal{H}_{-1}$ with respect to certain approximate solutions of Equation (1.2) in Theorem 2. The rigidity result could be expected for an elliptic equation. Note, however, that Equation (1.4) is a nonlinear hyperbolic equation. Since the definition of the so called branch points in [15] strongly used the explicit structure of Equation (1.1), it is not clear how to generalize Kirchheim's arguments to the perturbed case. We thus leave it as a topic to be studied in the near future.

The following theorem gives the main estimates in this paper.

THEOREM 1. Let $\Omega \subset \mathbb{R}^{2}$ be a bounded and convex Lipschitz domain. Assume $u \in W^{2, \infty}(\Omega)$ is a solution of Equation (1.4) with $\varphi$ satisfying

$$
\|\varphi-1\|_{W^{1, \infty}(\Omega)} \leq \delta<\frac{1}{2} .
$$

If we further assume

$$
\frac{\partial^{2} u}{\partial^{2} x_{1}} \leq-m^{2}, \frac{\partial^{2} u}{\partial^{2} x_{2}} \geq m^{2}, 0<m^{2}<\frac{1}{2},
$$


where $m$ is a nonzero constant, then for a given small enough $\varepsilon>0$ there exists a positive constant $\delta_{0}=\delta_{0}(\varepsilon, m, \operatorname{diam}(\Omega))$ such that the following holds:

if $\delta<\delta_{0}$, then there exist Bilipschitz maps $\widetilde{E} . \widetilde{R}$ and $D$ such that $\nabla D$ is diagonal, i.e.,

$$
\begin{gathered}
D\left(\begin{array}{l}
w_{1} \\
w_{2}
\end{array}\right)=\left(\begin{array}{l}
d_{1}\left(w_{1}\right) \\
d_{2}\left(w_{2}\right)
\end{array}\right), \\
\|\widetilde{E}-\mathrm{id}\|_{W^{1, \infty}(\Omega)}<\varepsilon,\|\widetilde{R}-\mathrm{id}\|_{W^{1, \infty}(\Omega)}<\varepsilon,
\end{gathered}
$$

and

$$
\widetilde{E}\left(\begin{array}{l}
\frac{\partial u}{\partial x_{1}}+x_{2} \\
\frac{\partial u}{\partial x_{1}}-x_{2}
\end{array}\right)=D \circ \widetilde{R}\left(\begin{array}{l}
\frac{\partial u}{\partial x_{2}}-x_{1} \\
\frac{\partial u}{\partial x_{2}}+x_{1}
\end{array}\right)
$$

This result immediately implies a rigidity estimate for compact perturbations of affine data.

COROLlary 1. Suppose all the hypotheses of Theorem 1 hold. If we further assume $\nabla^{2} u=A$ in a neighborhood of $\partial \Omega$, where $A$ is a constant matrix with $\operatorname{det} A=-1$, then

$$
\left\|\nabla^{2} u-A\right\|_{L^{\infty}(\Omega)}<\delta \cdot C(m, \operatorname{diam}(\Omega)) .
$$

More generally, we have the following rigidity result:

THEOREM 2.

(i) Suppose $\Omega$ is a bounded Lipschitz domain in $\mathbb{R}^{2}$, which is not necessarily simply connected, and $K$ is a compact subset of $\mathcal{H}_{-1}$ with sufficiently small diameter $\operatorname{diam}(K)$. Let $u^{(j)}$ be a sequence of functions in $W^{2, \infty}(\bar{\Omega})$ with

$$
\operatorname{dist}\left(\nabla^{2} u^{(j)}, K\right) \rightarrow 0 \text { in } W_{0}^{1, \infty}(\Omega)
$$

and on the boundary $\partial \Omega$,

$$
\nabla u^{(j)}\left\lfloor_{\partial \Omega}=F, \nabla^{2} u^{(j)}\left\lfloor_{\partial \Omega}=\nabla F=A,\right.\right.
$$

for some affine map $F$. Then,

$$
\nabla^{2} u^{(j)} \rightarrow A \text { in } L^{\infty}(\Omega)
$$

(ii) The restriction on $\operatorname{diam}(K)$ can be dropped if the sequence of functions are in $C^{2}(\bar{\Omega})$.

If $\Omega$ is simply connected, then the assumption in the first part of Equation (1.7), $\nabla u^{(j)}\left\lfloor_{\partial \Omega}=F\right.$, is redundant since the connectedness of $\partial \Omega$ implies the assumption up to a constant. 
ACKNOWLEDGEMENTS. I would like to thank Bernd Kirchheim for introducing the problem, Stefan Müller for revising the manuscript and improving the presentation of the proofs, and the Max Planck Institute for Mathematics in the Sciences, Leipzig/Germany for support during this research. In addition, I appreciate the referee for the comments and suggestions used to revise this paper, and Simon Morgan for his help in revising.

\section{2. - Proof of the main results}

To simplify our notation, it will be more convenient to establish the equivalent representative

$$
E\left(\begin{array}{c}
f_{1}(x) \\
x_{2}
\end{array}\right)=D \circ R\left(\begin{array}{c}
x_{1} \\
f_{2}(x)
\end{array}\right),
$$

with

$$
\left\|\nabla E-\left(\begin{array}{cc}
1 & 1 \\
1 & -1
\end{array}\right)\right\|_{L^{\infty}}<\varepsilon,\left\|\nabla R-\left(\begin{array}{cc}
-1 & 1 \\
1 & 1
\end{array}\right)\right\|_{L^{\infty}}<\varepsilon .
$$

The maps $E$ and $R$ will be constructed by Riemann invariants. The relation between the various maps and domains which appear in the proof is summarized in Figure 1. We remark that the map $\Sigma$ exists only when $f$ is invertible. We will, however, not use $\Sigma$ but work directly with $g$ and $h$.

$$
\begin{array}{ccccc}
\left(f_{1}, f_{2}\right) & \stackrel{\Sigma}{-\rightarrow} & \left(x_{1}, f_{2}\right) \in \mathcal{U}_{z} & \stackrel{R}{\longrightarrow} & \left(w_{1}, w_{2}\right) \in \mathcal{U}_{w} \\
f \uparrow & \nearrow & g \uparrow \downarrow h & \downarrow D \\
\left(x_{1}, x_{2}\right) \in \Omega & \stackrel{T}{\longrightarrow} & \left(f_{1}, x_{2}\right) \in \mathcal{U}_{\xi} & \stackrel{E}{\longrightarrow} & \left(\eta_{1}, \eta_{2}\right) \in \mathcal{U}_{\eta}
\end{array}
$$

Fig. 1. Outline of the changes of variables in the proof.

Proof of Theorem 1. We first study the geometry of the restrictions, from Equation (1.4) and (1.6), on $\nabla^{2} u$. The norm of a symmetric $2 \times 2$ matrix $A=\left(\begin{array}{ll}a_{11} & a_{12} \\ a_{21} & a_{22}\end{array}\right)$ is defined as

$$
\|A\|:=\sqrt{\frac{1}{2} \sum_{i, j=1}^{2} a_{i j}^{2}} .
$$

Applying the coordinates $\left(\begin{array}{cc}Z-X & Y \\ Y & Z+X\end{array}\right)$ on symmetric $2 \times 2$ matrices, we have the form: $\|A\|=\sqrt{X^{2}+Y^{2}+Z^{2}}$. Assume $Z_{0} \geq 0$ is a fixed constant. Then, 
Equation (1.6) means that $X$ satisfies both $X \geq m^{2}+Z_{0}$ and $X \geq m^{2}-Z_{0}$. Thus, $X \geq m^{2}+Z_{0}$. By Equation (1.4),

$$
0 \leq Y^{2}=Z_{0}^{2}-X^{2}+\varphi^{2} \leq Z_{0}^{2}+\varphi^{2}-\left(m^{2}+Z_{0}\right)^{2}=\varphi^{2}-2 m^{2} Z_{0}-m^{4}
$$

By Equation (1.5) and the same calculation in the case: $Z_{0}<0$, we conclude that

$$
\left|\frac{\partial^{2} u}{\partial x_{1} \partial x_{2}}\right| \leq \sqrt{\frac{1}{4}-m^{4}}
$$

Furthermore, $Z_{0}$ satisfies

$$
\left|Z_{0}\right| \leq \frac{\varphi^{2}-m^{4}}{2 m^{2}}
$$

Now, Equation (1.5) implies that $\operatorname{dist}\left(\nabla^{2} u, \mathcal{H}_{-1}\right)$ is finite in the sense of $L^{\infty}$ norm. Meanwhile, Equation (2.3) implies $\nabla^{2} u$ lies between the two hyperplanes, $\{(X, Y, Z): Z=c(m)\}$ and $\{(X, Y, Z): Z=-c(m)\}$. By the geometry of the hyperboloid $\mathcal{H}_{-1}$, we conclude that there exists a positive constant $M$, depending on $m$, such that

$$
\sup _{i, j, x}\left|\frac{\partial^{2} u(x)}{\partial x_{i} \partial x_{j}}\right| \leq M<\infty,
$$

For simplicity, we abbreviate $\frac{\partial^{2} u}{\partial x_{i}^{2}}$ and $\frac{\partial f_{j}}{\partial x_{i}}$ as $\partial_{i}^{2} u$ and $\partial_{i} f_{j}$ respectively in the rest of this paper.

STEP 1. (Existence of maps $g, h$ and the hyperbolic system for $g$ ).

Set $f(x)=\nabla u(x)$. Define the map $S: \Omega \rightarrow \mathbb{R}^{2}$ by

$$
S\left(x_{1}, x_{2}\right)=\left(x_{1}, f_{2}(x)\right) \text {. }
$$

Then $S$ is Lipschitz with

$$
\nabla S=\left(\begin{array}{cc}
1 & 0 \\
\partial_{1} f_{2} & \partial_{2} f_{2}
\end{array}\right), \operatorname{det} \nabla S=\partial_{2}^{2} u \geq m^{2}>0 .
$$

Thus $S$ is open and $\mathcal{U}_{z}:=S(\Omega)$ is open. Since $x_{2} \longmapsto f_{2}\left(x_{1}, x_{2}\right)$ is strictly monotone and $\Omega$ is convex, the map $S$ is injective and has a Lipschitz inverse $S^{-1}: \mathcal{U}_{z} \rightarrow \Omega$ with

$$
\left(\nabla S^{-1}\right) \circ S=\frac{1}{\partial_{2} f_{2}}\left(\begin{array}{cc}
\partial_{2} f_{2} & 0 \\
-\partial_{1} f_{2} & 1
\end{array}\right)
$$

Similarly the map

$$
T\left(x_{1}, x_{2}\right)=\left(f_{1}(x), x_{2}\right)
$$


is Bilipschitz with

$$
\nabla T=\left(\begin{array}{cc}
\partial_{1} f_{1} & \partial_{2} f_{1} \\
0 & 1
\end{array}\right),\left(\nabla T^{-1}\right) \circ T=\frac{1}{\partial_{1} f_{1}}\left(\begin{array}{cc}
1 & -\partial_{2} f_{1} \\
0 & \partial_{1} f_{1}
\end{array}\right),
$$

and

$$
\operatorname{det} \nabla T=\partial_{1}^{2} u \leq-m^{2}<0 .
$$

We set $\mathcal{U}_{\xi}:=T(\Omega)$ and define Bilipschitz maps

$$
g=S \circ T^{-1}, h=T \circ S^{-1}=g^{-1} .
$$

Then

$$
(\nabla g) \circ T=\frac{1}{\partial_{1} f_{1}}\left(\begin{array}{cc}
1 & -\partial_{2} f_{1} \\
\partial_{1} f_{2} & \operatorname{det} \nabla f
\end{array}\right) .
$$

Keeping in mind that $\partial_{1} f_{2}=\partial_{2} f_{1}$ and $\operatorname{det} \nabla f=-\varphi^{2}$, we deduce that for $g(\xi)$,

$$
\frac{\partial}{\partial \xi_{2}} g+\left(\begin{array}{cc}
0 & 1 \\
\varphi^{2}\left(z_{1}, \xi_{2}\right) & 0
\end{array}\right) \cdot \frac{\partial}{\partial \xi_{1}} g=0
$$

for $\xi \in T(\Omega)$ and $z=g(\xi)$. The matrix $\left(\begin{array}{cc}0 & 1 \\ \varphi^{2}\left(z_{1}, \xi_{2}\right) & 0\end{array}\right)$ has eigenvalues

$$
\lambda^{ \pm}= \pm \varphi\left(z_{1}, h_{2}(z)\right)
$$

with right and left eigenvectors

$$
r^{ \pm}=\left(1, \pm \varphi\left(z_{1}, h_{2}(z)\right)\right), l^{ \pm}=\left( \pm \varphi\left(z_{1}, h_{2}(z)\right), 1\right) .
$$

In the above, the notation \pm means either + or - . From now on, denote the opposite sign of \pm as $\mp$.

STEP 2 (Globally defined Riemann invariants and Bilipschitz maps $R$ and $E$ ).

For a fixed point $z^{(0)} \in \mathcal{U}_{z}$ and $\pm \in\{+,-\}$, we define the rarefaction curve $R^{ \pm}\left(z^{(0)}\right)$ locally in $\mathcal{U}_{z}$ by

$$
\left\{\begin{array}{l}
\frac{d}{d t} \alpha^{ \pm, z^{(0)}}(t)=\left(1, \pm \varphi\left(\alpha_{1}^{ \pm, z^{(0)}}(t), h_{2}\left(\alpha^{ \pm, z^{(0)}}(t)\right)\right)\right), \\
\alpha^{ \pm, z^{(0)}}(0)=z^{(0)}
\end{array}\right.
$$

By basic ODE theory, the Lipschitz condition of $\varphi$ and $h$ implies the existence of the Lipschitz solutions of Equation (2.10). We may assume $\left(c_{1}, c_{2}\right) \in \Omega$, for some constants $c_{1}$ and $c_{2}$. We may assume that the rarefaction curves $\cup_{z^{(0)}, \pm}\left\{R^{ \pm}\left(z^{(0)}\right)\right\}$ parametrize the domain $\mathcal{U}_{z}$, because of the local uniqueness of the solution of Equation (2.10). Since $\varphi \approx 1$, the curve $R^{ \pm}\left(z^{(0)}\right)$ intersects 
the line $\left\{z_{1}=c_{1}\right\}$ transversely. Define the Riemann invariants $w_{+}\left(z^{(0)}\right)$ and $w_{-}\left(z^{(0)}\right)$ by

$$
w_{ \pm}\left(z^{(0)}\right):=\left\{\widehat{z}_{2}: \text { if }\left(c_{1}, \widehat{z}_{2}\right) \text { lies on the curves } R^{ \pm}\left(z^{(0)}\right)\right\} .
$$

By the definition of $w_{ \pm}\left(z^{(0)}\right)$ and Equations (2.10), (2.9), we have

$$
\nabla_{z} w_{ \pm} \cdot r^{ \pm}=0, \forall \pm \in\{+,-\}
$$

Let $z^{(0)}=\left(z_{1}^{(0)}, z_{2}^{(0)}\right)$ and $\alpha^{ \pm, z^{(0)}}(t)$ be the solution of Equation (2.10). It is easy to verify that

$$
w_{ \pm}\left(z^{(0)}\right)=z_{2}^{(0)} \mp \int_{c_{1}}^{z_{1}^{(0)}} \varphi\left(t, h_{2}\left(\alpha^{ \pm, z^{(0)}}(t)\right)\right) d t
$$

From now on, we identify the notation $\left(w_{+}, w_{-}\right)$with $\left(w_{1}, w_{2}\right)$. Denote by $R$ the map $R(z)=w$ and the set $R\left(\mathcal{U}_{z}\right)$ by $\mathcal{U}_{w}$. Notice so far, the Riemann invariants $w_{ \pm}$are not globally defined on $\mathcal{U}_{z}$. This is because that the rarefaction curves $\alpha^{ \pm, z}(t)$ only exist in $\mathcal{U}_{z}$, thus may not intersect any of the lines $\left\{z_{i}=c_{i}\right\}$, $i \in\{1,2\}$. However, by Kirszbraun's Lipschitz extension theorem (see [10] 2.10.43), we can extend the Lipschitz function

$$
\psi(z):=\varphi\left(z_{1}, h_{2}(z)\right)
$$

from $\mathcal{U}_{z}$ to $\mathbb{R}^{2}$ such that $\bar{\psi}: \mathbb{R}^{2} \rightarrow \mathbb{R}$, with

$$
\operatorname{Lip}(\bar{\psi})=\operatorname{Lip}(\psi),\|\bar{\psi}-1\|_{L^{\infty}}<\delta \cdot\left(\operatorname{diam}\left(\mathcal{U}_{z}\right)+1\right)
$$

Replace the differential equation in Equation (2.10) by

$$
\frac{d}{d t} \alpha^{ \pm, z}(t)=\left(1, \pm \bar{\psi}\left(\alpha^{ \pm, z}(t)\right)\right)
$$

then the rarefaction curves $\alpha^{ \pm, z}(t)$ are globally defined on $\mathbb{R}^{2}$, the $z$-plane. Hence, the Riemann invariants $w_{ \pm}$are well defined on $\mathcal{U}_{z}$.

Now, we show that the map $R$ is Bilipschitz. From Equations (2.11) and (2.13), for any $z^{(0)}, z^{(1)} \in \mathcal{U}_{z}$, we have

$$
\begin{aligned}
w^{ \pm}\left(z^{(1)}\right)-w^{ \pm}\left(z^{(0)}\right) & =\left(z_{2}^{(1)}-z_{2}^{(0)}\right) \mp\left(z_{1}^{(1)}-z_{1}^{(0)}\right) \\
& \mp \int_{z_{1}^{(1)}}^{z_{1}^{(1)}}\left[\bar{\psi}\left(\alpha^{ \pm, z^{(1)}}(t)\right)-1\right] d t \\
& \mp \int_{c_{1}}^{z_{1}^{(0)}}\left[\bar{\psi}\left(\alpha^{ \pm, z^{(1)}}(t)\right)-\bar{\psi}\left(\alpha^{ \pm, z^{(0)}}(t)\right)\right] d t .
\end{aligned}
$$


By Equation (1.5),

$$
\left|\int_{z_{1}^{(0)}}^{z_{1}^{(1)}}\left[\bar{\psi}\left(\alpha^{ \pm, z^{(1)}}(t)\right)-1\right] d t\right|<\delta \cdot\left(\operatorname{diam}\left(\mathcal{U}_{z}\right)+1\right) \cdot\left|z^{(1)}-z^{(0)}\right| .
$$

By Equations (2.2), (1.6), (2.5), (2.6), (2.7), (2.8), (2.12) we have

$$
\begin{aligned}
\operatorname{diam}\left(\mathcal{U}_{z}\right) & \leq \sup |\nabla S| \cdot \operatorname{diam}(\Omega) \leq C(m) \cdot \operatorname{diam}(\Omega), \\
\operatorname{Lip}(\bar{\psi}) & =\operatorname{Lip}(\psi) \leq \operatorname{Lip}(\varphi) \cdot \operatorname{Lip}\left(S^{-1}\right) \leq \operatorname{Lip}(\varphi) \cdot C(m),
\end{aligned}
$$

and

$$
\begin{aligned}
\frac{1}{\left|z^{(1)}-z^{(0)}\right|} \mid & \int_{c_{1}}^{z_{1}^{(0)}}\left[\bar{\psi}\left(\alpha^{ \pm, z^{(1)}}(t)\right)-\bar{\psi}\left(\alpha^{ \pm, z^{(0)}}(t)\right)\right] d t \mid \\
& \leq \operatorname{diam}\left(\mathcal{U}_{z}\right) \cdot \operatorname{Lip}(\bar{\psi}) \sup _{t} \frac{\left|\alpha^{ \pm, z^{(1)}}(t)-\alpha^{ \pm, z^{(0)}}(t)\right|}{\left|z^{(1)}-z^{(0)}\right|} .
\end{aligned}
$$

By standard ODE theory, we have

$$
\lim _{z^{(1)} \rightarrow z^{(0)}}\left(\sup _{t} \frac{\left|\alpha^{ \pm, z^{(1)}}(t)-\alpha^{ \pm, z^{(0)}}(t)\right|}{\left|z^{(1)}-z^{(0)}\right|}\right) \leq \exp \left(\operatorname{Lip}(\bar{\psi}) \cdot \operatorname{diam}\left(\mathcal{U}_{z}\right)\right) .
$$

By the definition of the map $R$ and the estimates in Equations (2.14) (2.18), one can verify that we have the estimate,

$$
\left\|\nabla R-\left(\begin{array}{cc}
-1 & 1 \\
1 & 1
\end{array}\right)\right\|_{L^{\infty}\left(\overline{\mathcal{U}_{z}}\right)}<\delta \cdot C_{1}(m, \operatorname{diam}(\Omega)) .
$$

If we assume $\varepsilon=\delta \cdot C_{1}$ is sufficiently small, then $R$ is Bilipschitz. By standard ODE theory, we have the local existence and uniqueness for the solutions of Equation (2.10). This implies that $R$ is injective. Thus $R^{-1}$ exists and we may treat $\xi$ as the function of $w$ by setting $\xi=h \circ R^{-1}(w)$.

Step 3 (Properties of the diagonal map $D$ between two pairs of Riemann invariants).

For fixed $\xi^{(0)}:=h\left(z^{(0)}\right)$ and $\pm \in\{+,-\}$, denote the Lipschitz curve $h \circ \alpha^{ \pm, z^{(0)}}(t)$ in $\mathcal{U}_{\xi}$ as $E_{ \pm}\left(\xi^{(0)}\right)$. Let

$$
\beta^{ \pm, \xi^{(0)}}(t):=h \circ \alpha^{ \pm, z^{(0)}}(t) .
$$

Then, by Equations (2.7), (2.8), and (2.10), one can verify that

$$
\frac{d}{d t} \beta^{ \pm, \xi^{(0)}}(t)=(\nabla h)\left(\alpha^{ \pm, z^{(0)}}(t)\right) \cdot \frac{d}{d t} \alpha^{ \pm, z^{(0)}}(t)=\frac{ \pm \varphi-\partial_{1} f_{2}}{\partial_{2} f_{2}} \cdot\left(\begin{array}{c}
\mp \varphi \\
1
\end{array}\right)
$$


The assumptions in Equations (1.5), (2.2) and (1.6) imply that $\frac{ \pm \varphi-\partial_{1} f_{2}}{\partial_{2} f_{2}}$ is bounded, nonzero and stays one-signed. One can verify that the Lipschitz curve $E^{ \pm}\left(\xi^{(0)}\right)$ can be described by $\gamma^{ \pm, \xi^{(0)}}(s)$, which satisfies

$$
\left\{\begin{array}{l}
\frac{d}{d s} \gamma^{ \pm, \xi^{(0)}}(s)=\left(\mp \varphi\left(g_{1}\left(\gamma^{ \pm, \xi^{(0)}}(s)\right), s\right), 1\right), \\
\gamma^{ \pm, \xi^{(0)}}(0)=\xi^{(0)}
\end{array}\right.
$$

Define the quantity $\eta_{ \pm}$(or called the Riemann invariant) on $\mathcal{U}_{\xi}$ by

$$
\eta_{ \pm}\left(\xi^{(0)}\right):=\left\{\widehat{\xi}_{1}:\left(\widehat{\xi}_{1}, c_{2}\right) \text { lies on the curves } E^{ \pm}\left(\xi^{(0)}\right)\right\}
$$

It is easy to verify that

$$
\eta_{ \pm}\left(\xi^{(0)}\right)=\xi_{1}^{(0)} \pm \int_{c_{2}}^{\xi_{2}^{(0)}} \varphi\left(g_{1}\left(\gamma^{ \pm, \xi^{(0)}}(s)\right), s\right) d s
$$

By a similar argument as in Step $2^{0}$, one can verify that the map $E$ is well defined on $\mathcal{U}_{\xi}$ and Bilipschitz with

$$
\left\|\nabla E-\left(\begin{array}{cc}
1 & 1 \\
1 & -1
\end{array}\right)\right\|_{L^{\infty}\left(\overline{\mathcal{U}_{\xi}}\right)}<\delta \cdot C_{1}(m, \operatorname{diam}(\Omega))
$$

The Bilipschitz map $R$ maps the rarefaction curves $\alpha^{ \pm}$to the lines $\left\{w_{ \pm}=\right.$ constant. Similarly, $E$ maps the curves $\beta^{ \pm}=h \circ \alpha^{ \pm}$to the lines $\left\{\eta_{ \pm}=\right.$ constant. Hence $D:=E \circ h \circ R^{-1}$ is Bilipschitz which maps $\left\{w_{1}=\right.$ constant to $\left\{\eta_{1}=\right.$ constant and $\left\{w_{2}=\right.$ constant to $\left\{\eta_{2}=\right.$ constant. Therefore, $D(w)=$ $\left(d_{1}\left(w_{1}\right), d_{2}\left(w_{2}\right)\right)$ and $E \circ h=D \circ R$. Recall the definition of $h$, we find $\eta=D(w)$ on $\mathcal{U}_{w}$ and

$$
\nabla D=\left(\begin{array}{ll}
\frac{\partial \eta_{1}}{\partial w_{1}} & \frac{\partial \eta_{1}}{\partial w_{2}} \\
\frac{\partial \eta_{2}}{\partial w_{1}} & \frac{\partial \eta_{2}}{\partial w_{2}}
\end{array}\right)=\left(\begin{array}{cc}
\dot{d}_{1}\left(w_{1}\right) & 0 \\
0 & \dot{d}_{2}\left(w_{2}\right)
\end{array}\right),
$$

where $\dot{d}_{i}$ denotes the derivative of the functions of one-variable $d_{i}$.

Let $\widetilde{R}:=R \circ L_{z}$ and $\widetilde{E}:=E \circ L_{\xi}$, where

$$
\begin{gathered}
L_{z}:\left(\begin{array}{l}
z_{1} \\
z_{2}
\end{array}\right) \mapsto\left(\begin{array}{c}
\left(z_{2}-z_{1}\right) / 2 \\
\left(z_{2}+z_{1}\right) / 2
\end{array}\right), \\
L_{\xi}:\left(\begin{array}{l}
\xi_{1} \\
\xi_{2}
\end{array}\right) \mapsto\left(\begin{array}{l}
\left(\xi_{1}+\xi_{2}\right) / 2 \\
\left(\xi_{1}-\xi_{2}\right) / 2
\end{array}\right) .
\end{gathered}
$$

Then the proof is finished. 
Proof of Corollary 1. From the proof in Theorem 1, it is obvious that for each fixed $x^{(0)} \in \Omega$ there exist two points $x^{(+)}, x^{(-)}$on $\partial \Omega$ such that $S\left(x^{( \pm)}\right) \in \alpha^{ \pm, S\left(x^{(0)}\right)}$. Since $\frac{ \pm \varphi-\partial_{1} f_{2}}{\partial_{2} f_{2}}$ is bounded and stays one-signed, one can simply verify that the approximate tangent lines of the Lipschitz curve $S^{-1}\left(\alpha^{ \pm, S\left(x^{(0)}\right)}\right)$ are never vertical, horizontal or changing the sign of slope in the chosen coordinates of $\Omega$. From Figure 1 and Theorem 1, one can further verify that

$$
\left\|\nabla D \circ R \circ S-\left(\begin{array}{cc}
\frac{\partial_{1} f_{2}+1}{\partial_{2} f_{2}} & 0 \\
0 & \frac{\partial_{1} f_{2}-1}{\partial_{2} f_{2}}
\end{array}\right)\right\|_{L^{\infty}(\Omega)}<\delta \cdot C_{2}(m, \operatorname{diam}(\Omega)) .
$$

By Equations (2.23), (2.24) and triangle inequality, we obtain the estimates

$$
\left|\frac{\partial_{1} f_{2} \pm 1}{\partial_{2} f_{2}}\left(x^{(0)}\right)-\frac{\partial_{1} f_{2} \pm 1}{\partial_{2} f_{2}}\left(x^{(\mp)}\right)\right|<\delta \cdot 2 C_{2}(m, \operatorname{diam}(\Omega)),
$$

for each $\pm \in\{+,-\}$. The proof is then finished by applying the affine boundary condition.

Proof of Theorem 2. (i) For each $f^{(j)}$, we want to apply the argument in the proof of Theorem 1 and Corollary 1. Thus, we need to check if the slightly different assumptions in Theorem 2 would cause problems. When $j$ is sufficiently large and $\operatorname{diam}(K)$ is sufficiently small, the assumptions in Equation (2.2) and (1.6) are fulfilled by a suitable rotation of coordinates in $\Omega$. Notice that, here, we apply the property: both $\left\|\nabla^{2} u\right\|$ and $\operatorname{det} \nabla^{2} u$ are invariant under rotations of the coordinates of $\Omega$. Due to the nonlinear change of variables associated to $f^{(j)}$, we have the maps $S_{j}$ and $T_{j}$, which denote the maps $S$ and $T$ respectively in Figure 1. However, without assuming the convexity of $\Omega$, the inverse map $S_{j}^{-1}$ or $T_{j}^{-1}$ may not exist. This problem can be fixed by the affine boundary condition in Equation (1.7). Namely, we have the Lipschitz extension of $f^{(j)}$ by defining

$$
\bar{f}^{(j)}(x):= \begin{cases}f^{(j)}(x), & x \in \Omega, \\ F(x), & x \in \mathbb{R}^{2} \sim \Omega .\end{cases}
$$

Since $\nabla \bar{f}^{(j)}$ is symmetric and uniformly bounded on $\mathbb{R}^{2}$, there exists an entire function $\bar{u}^{(j)}$ so that $\nabla \bar{u}^{(j)}=\bar{f}^{(j)}$. It is easy to verify that $\bar{u}^{(j)}$ satisfies the assumptions in Equations (1.5), (2.2), (1.6), and thus both $\partial_{2}^{2} \bar{u}^{(j)}$ and $\partial_{1}^{2} \bar{u}^{(j)}$ are strictly positive and negative. This guarantees the existence of both the inverse maps of $\overline{S_{j}}$ and $\overline{T_{j}}: \mathbb{R}^{2} \rightarrow \mathbb{R}^{2}$, which are maps induced from $\nabla \bar{u}^{(j)}$ as shown in Figure 1. Therefore, both the inverse maps $S_{j}^{-1}$ and $T_{j}^{-1}$ exist.

The proof is finished by following the argument in Theorem 1 and Corollary 1. 
(ii) Since $\Omega$ is bounded, we can choose a domain of rectangle in $\mathbb{R}^{2}$ to contain $\Omega$. Namely, for each fixed $k \in\{1,2\}$, we can define the finite numbers,

$$
x_{k}^{\mathrm{inf}}(\Omega):=\inf _{x \in \Omega}\left\{x_{k}: x=\left(x_{1}, x_{2}\right) \in \Omega\right\} ; x_{k}^{\text {sup }}(\Omega):=\sup _{x \in \Omega}\left\{x_{k}: x=\left(x_{1}, x_{2}\right) \in \Omega\right\} .
$$

Thus, $\Omega \subset\left[x_{1}^{\inf }(\Omega), x_{1}^{\text {sup }}(\Omega)\right] \times\left[x_{2}^{\inf }(\Omega), x_{2}^{\text {sup }}(\Omega)\right]$. Let $t>0$ and define the domains

$$
\Omega_{t}:=\left\{x=\left(x_{1}, x_{2}\right) \in \Omega: x_{2}>x_{2}^{\text {sup }}(\Omega)-t\right\} .
$$

By choosing a subsequence, we may suppose as $j \rightarrow \infty, \operatorname{dist}\left(\nabla^{2} u^{(j)}, K\right)$ decreases monotonically in the sense of $W^{1, \infty}(\bar{\Omega})$. For given $\varepsilon>0$ and $j$, let

$$
V_{j}^{\varepsilon}:=\left\{x \in \Omega:\left\|\nabla^{2} u^{(j)}-A\right\|_{L^{\infty}(\Omega)}<\varepsilon\right\} .
$$

Since $u^{(j)} \in C^{2}(\bar{\Omega}), V_{j}^{\varepsilon}$ is an open set. Let

$$
t_{j}^{\varepsilon}:=\sup \left\{t: \Omega_{t} \subset V_{j}^{\varepsilon}\right\} \text {, and } \mathcal{I}_{j}^{\varepsilon}:=\left(0, t_{j}^{\varepsilon}\right) \subset(0, \infty) .
$$

Now we claim: for given $\varepsilon>0$, there exists $J^{\varepsilon} \in \mathbb{N}$ such that

$$
\left\|\nabla^{2} u^{(j)}-A\right\|_{L^{\infty}(\Omega)}<\varepsilon, \text { if } j \geq J^{\varepsilon} .
$$

This is equivalent to showing that for fixed $\varepsilon>0, \mathcal{I}_{j}^{\varepsilon}=(0, \infty)$ for some sufficiently large $j$. Since for each $j, u^{(j)} \in C^{2}(\bar{\Omega})$, thus $\mathcal{I}_{j}^{\varepsilon}$ is open and nonempty. Therefore, we only need to show that for some sufficiently large $j, \mathcal{I}_{j}^{\varepsilon}$ is also closed in $(0, \infty)$. Assume on the contrary that $\sup _{j} t_{j}^{\varepsilon} x_{2}^{\text {sup }}(\Omega)-x_{2}^{\text {inf }}(\Omega)$. In other words, for any sufficiently large $j$, there exists $a^{(j)}=\left(a_{1}^{(j)}, a_{2}^{(j)}\right) \in \Omega$ with $a_{2}^{(j)}=x_{2}^{\text {sup }}(\Omega)-t_{j}^{\varepsilon}$ such that

$$
\left\|\nabla^{2} u^{(j)}\left(a^{(j)}\right)-A\right\|_{L^{\infty}(\Omega)} \geq \varepsilon .
$$

Since the slope of the curve $S^{-1}\left(\alpha^{ \pm, S\left(a^{(j)}\right)}\right)$ is one-signed for each $\pm \in\{+,-\}$, the curve $S^{-1}\left(\alpha^{ \pm, S\left(a^{(j)}\right)}\right)$ must intersect $\partial \Omega_{t_{j}^{\varepsilon}} \sim\left\{\left(x^{1}, x^{2}\right) \in \Omega: x_{2}=t_{j}^{\varepsilon}\right\}$, which is a subset of $\partial \Omega$.

Now, applying the argument in Theorem 2 (i) on $\Omega_{t_{j}^{\varepsilon}}$, we obtain contradiction from the conclusion of Theorem 2 i).

\section{REFERENCES}

[1] R. Aumann - S. Hart, Bi-convexity and bi-martingales, Israel J. Math. 54 (1986), 159180.

[2] J. M. BALL - R. D. JAMES, Fine phase mixtures as minimizers of energy, Arch. Ration. Mech. Anal. 100 (1987), 13-52. 
[3] E. Casadio-Tarabusi, An algebraic characterization of quasi-convex functions, Ricerche Mat. 42 (1993), 11-24.

[4] N. Chaudhuri - S. Müller, Rank-one convexity implies quasiconvexity on certain hypersurfaces, Proc. Roy. Soc. Edinburgh Sect. A 133 (2003), 1263-1272.

[5] M. Chlebík - B. Kirchneim, Rigidity for the four gradient problem, J. Reine Angew. Math. 551 (2002), 1-9.

[6] M. Chipot - D. Kinderlehrer, Equilibrium configurations of crystals, Arch. Ration. Mech. Anal. 103 (1988), 237-277.

[7] G. Dolzmann, "Variational Methods for Crystalline Microstructure-Analysis and Computation”, Springer Lecture Notes in Mathematics 1803, 2003.

[8] L. C. Evans, "Partial differential equations", Graduate Studies in Mathematics, 19, American Mathematical Society, Providence, RI, 1998.

[9] L. C. Evans - R. F. GARIEPY, On the partial regularity of energy-minimizing, areapreserving maps, Calc. Var. Partial Differential Equations (4) 9 (1999), 357-372.

[10] H. Federer, "Geometric Measure Theory”, Springer-verlag, New York, 1969.

[11] G. FRIESECKE - R. D. JAMES - S. MüLleR, A theorem on geometric rigidity and the derivation of nonlinear plate theory from three-dimensional elasticity, Comm. Pure Appl. Math. 55 (2002), 1461-1506.

[12] E. HEINZ, Über die Lösungen der Minimalfä chengleichung, Nach. Akad. Wissensch. in Göttingen Math.-Phys. K1. II (1952), 51-56.

[13] F. John, Bounds for deformations in terms of average strains, Inequalities, III (Proc. Third Sympos., Univ. California, Los Angeles, Calif., 1969; dedicated to the memory of Theodore S. Motzkin), pp. 129-144, Academic Press, New York, 1972.

[14] D. Kinderlehrer, Remarks about equilibrium configurations of crystals, in: "Material instabilities in continuum mechanics and related mathematical problems" J.M. Ball (eds.), Oxford University Press, 1988, pp. 217-242.

[15] B. Kirchneim, "Habilitation thesis", University of Leipzig, 2001.

[16] O. I. Мокноv - Y. Nutкu, Bianchi transformation between the real hyperbolic MongeAmpère equation and the Born-Infeld equation, Lett. Math. Phys. (2) 32 (1994), 121-123.

[17] S. MülLER, Variational models for microstructure and phase transitions, in: "Calculus of variations and geometric evolution problems" (Cetraro, 1996 eds.), pp. 85-210, Lecture Notes in Math., 1713, Springer, Berlin, 1999.

[18] S. MüLlER, Rank-one convexity implies quasiconvexity on diagonal matrices, Internat. Math. Res. Notices (20) (1999), 1087-1095.

[19] S. MÜLLER - V. ŠVERÁK, Convex integration for Lipschitz mappings and counterexamples to regularity, Ann. of Math. (2-3) 157 (2003), 715-742.

[20] V. NesI - G.W. Milton, Polycrystalline configurations that maximize electrical resistivity, J. Mech. Phys. Solids (4) 39 (1991), 525-542.

[21] V. Scheffer, "Regularity and irregularity of solutions to nonlinear second order elliptic systems of partial differential equations and inequalities", Dissertation, Princeton University, 1974.

[22] R. Schoen - J. Wolfson, Minimizing volume among Lagrangian submanifolds, Differential equations: La Pietra 1996, Florence, pp. 181-199, Proc. Sympos. Pure Math., 65, Amer. Math. Soc., Providence, RI, 1999. 
[23] F. Schulz, "Regularity theory for quasilinear elliptic systems and Monge-Amper̀e equations in two dimensions", Lecture Notes in Mathematics, 1445, Springer-Verlag, Berlin, 1990.

[24] V. ŠverÁk, "On regularity for the Monge-Ampère equations", preprint, Heriot-Watt University, 1991.

[25] L. TARTAR, Some remarks on separately convex functions, in: "Microstructure and phase transitions", IMA Vol. Math. Appl. 54 (D. Kinderlehrer, R. D. James, M. Luskin and J.L. Ericksen, eds.), Springer, 1993, pp. 191-204.

Department of Mathematics National Taiwan Normal University Taipei 116, Taiwan

chunlin@math.ntnu.edu.tw 